\title{
Efficient ferromagnetic core impedance model with application to finite-difference time-domain simulation
}

\author{
T. C. Genoni, D. V. Rose, R. E. Clark, and D. R. Welch \\ Voss Scientific, LLC, Albuquerque, New Mexico 87108, USA \\ W. A. Stygar \\ Sandia National Laboratories, Albuquerque, New Mexico 87185, USA
}

(Received 4 October 2011; published 9 January 2012)

\begin{abstract}
A frequency-dependent impedance model for laminated ferromagnetic cores is presented and analyzed. The model assumes a multiple-winding ferromagnetic induction core composed of multiple thin layers with linear material response. This model builds on the analysis presented by Rose et al. [Phys. Rev. ST Accel. Beams 13, 090401 (2010)], that determined an equivalent time-dependent resistance that was used to successfully model the loss currents in a linear transformer device cavity containing ferromagnetic cores. The new core impedance model is more general and has been implemented as a surface-impedance boundary condition [K. S. Oh and J. E. Schutt-Aine, IEEE Trans. Antennas Propag. 43, 660 (1995)] which is suitable for use in multidimensional finite-difference time-domain codes.
\end{abstract}

\section{INTRODUCTION}

Linear transformer drivers (LTDs) are a rapidly developing area of pulsed-power technology capable of delivering high-power, high-current, 100-300 ns output pulses in a compact configuration [1-6]. The LTD platform is a type of induction accelerator that utilizes close packing of primary energy storage and switching to achieve a shortduration, high-peak-power pulse. A single LTD cavity is comprised internally of capacitors, switches, and ferromagnetic cores. Within each cavity, a set of two capacitors and a switch, connected in series, is referred to as a brick. Individual bricks are typically arranged azimuthally around the inside of the cavity and are connected in parallel to a radial transmission line inside the cavity. The parallel plate radial transmission line is either directly connected to a load or (more typically) feeds a coaxial transmission line that joins many similar LTD cavities in serial to form a type of induction voltage adder [7]. Ferromagnetic cores inhibit the flow of current along the inside of the LTD cavity. These cores are constructed from a continuous winding of thin (50-100 $\mu \mathrm{m}$ thick) ferromagnetic material in a ring with the individual layers separated by a thin dielectric material. These cores are critical components of LTD cavities as well as other accelerator architectures [8].

New accelerators utilizing multiple LTD cavities are presently being developed for a number of pulsed-power and high-energy-density physics applications [9]. Triggering these LTD cavities at different times allows

Published by the American Physical Society under the terms of the Creative Commons Attribution 3.0 License. Further distribution of this work must maintain attribution to the author(s) and the published article's title, journal citation, and DOI. for precise shaping of the output power pulse [10,11]. Recently, electromagnetic (EM), finite-difference timedomain (FDTD) simulations have been used to model high-power LTD cavities [11]. These simulations provide valuable insight into the operation of an LTD cavity, including an assessment of EM field stresses that develop at critical component connections inside a cavity. The magnitudes of these effects can be greatly increased in multiple cavity accelerators, particularly when power-pulse shaping is invoked since EM waves entering untriggered cavities can create dynamic field stresses on critical electrical components.

In this paper we present a series of models for the equivalent impedance of the ferromagnetic cores used in high-power LTD systems in the production of submicrosecond time scale power pulses. The basis for the models was developed by Lammeraner and Stafl [12] and Grandi et al. [13], in which the frequency-dependent impedance of a nonsaturated laminated iron-core inductor was calculated using a one-dimensional analysis of eddy currents and field penetration and neglecting hysteresis effects. The physical representation for the ferromagnetic core described in this paper is illustrated in Fig. 1. A transmission line driven by a time-dependent voltage $V(t)$ results in current flow along the conducting path that encloses a volume containing a finite number of ferromagnetic slabs of thickness $\delta$ (where $\delta>\delta_{\omega}$, the electromagnetic skin depth) and permeability $\mu\left(\mu>\mu_{0}\right.$, the permeability of free space). Eddy currents, induced by the magnetic field of the current flowing along the cavity, flow along the slab surfaces. The magnetic field that diffuses into the core material is an energy sink.

The models presented here complement other recent analyses of LTD cores including Refs. [11,14]. The equivalent impedance model developed in Refs. [12,13] for a 




FIG. 1. Schematic of a core showing slabs of finite thickness $\delta$ and magnetic permeability $\mu$. Current flow along conductor enclosing the slabs is driven by a parallel plate transmission line with a gap spacing $h$. (This schematic is based on Fig. 2 of Ref. [14].)

laminated ferromagnetic core is reviewed in Sec. II. This model is used in the analysis of a transmission line terminated by the (time-dependent) core impedance in Sec. III. Numerical examples demonstrating the use of the transmission line model for different current waveforms are given. In particular, we demonstrate the equivalence of this model to the time-dependent core resistance model of Ref. [11] for the case of a linearly rising current waveform. In Sec. IV, we review the implementation of a surface-impedance boundary condition (SIBC) [15] for use in EM FDTD simulations. A new set of model coefficients, used by the SIBC algorithm and presented in Sec. IV, enables the use of this algorithm for frequency regimes appropriate for the analysis of ferromagnetic cores. In Sec. V, we show how the fit to the impedance function can be used to infer an equivalent circuit model for the ferromagnetic core. In Sec. VI, we compare these models with 2D EM simulations of an idealized ferromagnetic core that includes finite thickness slabs of linear media with prescribed conductivity and permeability. While these idealized simulations represent only a small spatial fraction of a core that is typically used in pulsedpower applications, they demonstrate the applicability of the SIBC to model the time-dependent response of the core in large-scale FDTD simulations. A summary of this work is given in Sec. VII.

\section{EQUIVALENT IMPEDANCE MODEL}

We consider the frequency response of the core assuming that the core material properties are independent of frequency (linear media) and that no hysteresis effects are present (i.e., core saturation is not modeled). Under these assumptions, Lammeraner and Stafl [12] and later Grandi et al. [13] developed the following approximate formula for the frequency-dependent impedance of a ferromagnetic inductor using a one-dimensional analysis of eddy currents and field penetration:

$$
Z_{c}(\omega)=i \omega L_{(\mathrm{dc})} \frac{2}{1+i} \frac{\delta_{\omega}}{\delta} \tanh \left(\frac{1+i}{2} \frac{\delta}{\delta_{\omega}}\right),
$$

where $\delta$ is the material thickness and $\omega$ is the angular frequency. The skin depth is

$$
\delta_{\omega}=\sqrt{\frac{2 \rho}{\mu \omega}},
$$

where $\mu$ and $\rho$ are the material permeability and resistivity, respectively. The dc inductance is defined as

$$
L_{(d c)}=\frac{\mu A}{\ell}=\frac{\mu n q \delta}{\ell},
$$

where (see Fig. 1) $q$ is the sheet width, $\ell$ is the sheet length, and $A=n q \delta$ is the total cross-sectional area of the $n$ laminations. Equation (1) may be cast in the form

$$
Z_{c}(s)=\frac{2 \sqrt{\mu \rho} A}{\ell \delta} \sqrt{s} \tanh \left(\sqrt{\frac{s}{a}}\right)
$$

where $s(=i \omega)$ is the Laplace transform variable. The frequency parameter $a$ is given by

$$
a=\frac{4 \rho}{\mu \delta^{2}} \text {. }
$$

\section{TRANSMISSION LINE ANALYSIS}

We consider the 2D transmission line and iron-core inductor geometry of Fig. 1 with the following parameters: $n=5, h=24 \mu \mathrm{m}, q=500 \mu \mathrm{m}$, and laminate thickness $\delta=50 \mu \mathrm{m}$. We leave the length $\ell$ unspecified and give currents in units of $\mathrm{kA} / \mathrm{cm}$. (These particular parameters are motivated by the 2D EM FDTD simulations described in Sec. VI.) For the numerical calculations below, we set $\mu=80 \mu_{0}$ and resistivity $\rho=10^{-7} \Omega \mathrm{m}$.

The response of the core is found by terminating the transmission line with the impedance of Eq. (4). For a voltage time history $V_{0}(t)$ with Laplace transform $\tilde{V}_{0}(s)$, standard transmission line analysis (neglecting very small transit times) gives the following expressions for the voltage and current per unit length at the termination impedance:

$$
\begin{gathered}
V_{c}(t)=\mathcal{L}^{-1}\left\{\tilde{V}_{0}(s) \frac{2 Z_{c}(s)}{\left[Z_{0}+Z_{c}(s)\right]}\right\}, \\
\frac{I_{c}(t)}{\ell}=\frac{1}{Z_{0}} \mathcal{L}^{-1}\left\{\tilde{V}_{0}(s) \frac{2 Z_{0}}{\left[Z_{0}+Z_{c}(s)\right]}\right\},
\end{gathered}
$$

where $Z_{0} / \ell=\mu_{0} c h / \ell$ is the impedance of the vacuum transmission line (in $\Omega$ ), and $\mathcal{L}^{-1}$ denotes inverse Laplace transformation. 
We consider first a power law voltage ramp of the form

$$
V_{0}(t)=\Delta V\left(\frac{t}{t_{0}}\right)^{k}, \quad k>0,
$$

with Laplace transform

$$
\tilde{V}_{0}(s)=\Delta V \frac{\Gamma(k+1)}{t_{0}^{k}} \frac{1}{s^{k+1}},
$$

where $t_{0}$ is a characteristic time scale. Substitution into Eqs. (6) and (7) gives

$$
\begin{gathered}
V_{c}(t)=\frac{\Delta V}{t_{0}} \mathcal{L}^{-1}\left\{\frac{\Gamma(k+1)}{t_{0}^{k-1}} \frac{1}{s^{k+1}} \frac{2 Z_{c}(s)}{\left[Z_{0}+Z_{c}(s)\right]}\right\}, \\
\frac{I_{c}(t)}{\ell}=\frac{\Delta V}{t_{0}} \frac{1}{Z_{0}} \mathcal{L}^{-1}\left\{\frac{\Gamma(k+1)}{t_{0}^{k-1}} \frac{1}{s^{k+1}} \frac{2 Z_{0}}{\left[Z_{0}+Z_{c}(s)\right]}\right\} .
\end{gathered}
$$

The inverse Laplace transforms in Eqs. (10) and (11) may be evaluated by the standard inversion formula and direct numerical integration along an appropriate contour in the complex plane. Carrying out this procedure for a linear voltage ramp $(k=1)$ of $\Delta V / t_{0}=10 \mathrm{kV} / \mathrm{ns}$ and the geometry and material parameters specified above produced the voltage and current time histories shown in Fig. 2.

Before we consider a more general voltage time history, we touch base with an approximate formula derived previously in Ref. [11] which applies to a linear ramp voltage drive. We first note that for times less than a few tens of nanoseconds we can ignore the low frequency contribution to $Z_{c}(s)$ and take $\tanh (\sqrt{s / a}) \simeq 1$ in Eq. (4). We also note that except for the very earliest times (highest frequencies) we may make the approximation $Z_{c}(s) \ll Z_{0}$ in Eqs. (10) and (11). The resulting Laplace transforms may then be inverted analytically to give

$$
R(t)=\frac{V_{c}(t)}{I_{c}(t)}=\frac{2 \sqrt{\mu \rho} A}{\ell \delta \sqrt{t}} \frac{\Gamma(k+1)}{\Gamma(k+1 / 2)},
$$

which should be valid over some intermediate range of times which will depend on the value of the parameter $a$ and the impedance of the transmission line. For $k=1$ (linear ramp), $\Gamma(k+1)=1$ and $\Gamma(k+1 / 2)=\sqrt{\pi} / 2$, and Eq. (12) reduces to

$$
R(t) \simeq \frac{4 \sqrt{\mu \rho} A}{\sqrt{\pi} \ell \delta \sqrt{t}}
$$

which is in agreement with Eq. (A8) of Ref. [11]. $R(t)$, for the $\Delta V / t_{0}=10 \mathrm{kV} / \mathrm{ns}$ linear ramp and full numerical Laplace transform inversion of Eqs. (10) and (11), is plotted in Fig. 3, along with the approximate formula of Eq. (13). Agreement is seen to be excellent down to a few tenths of a nanosecond.

We also considered a second voltage drive time history of the form
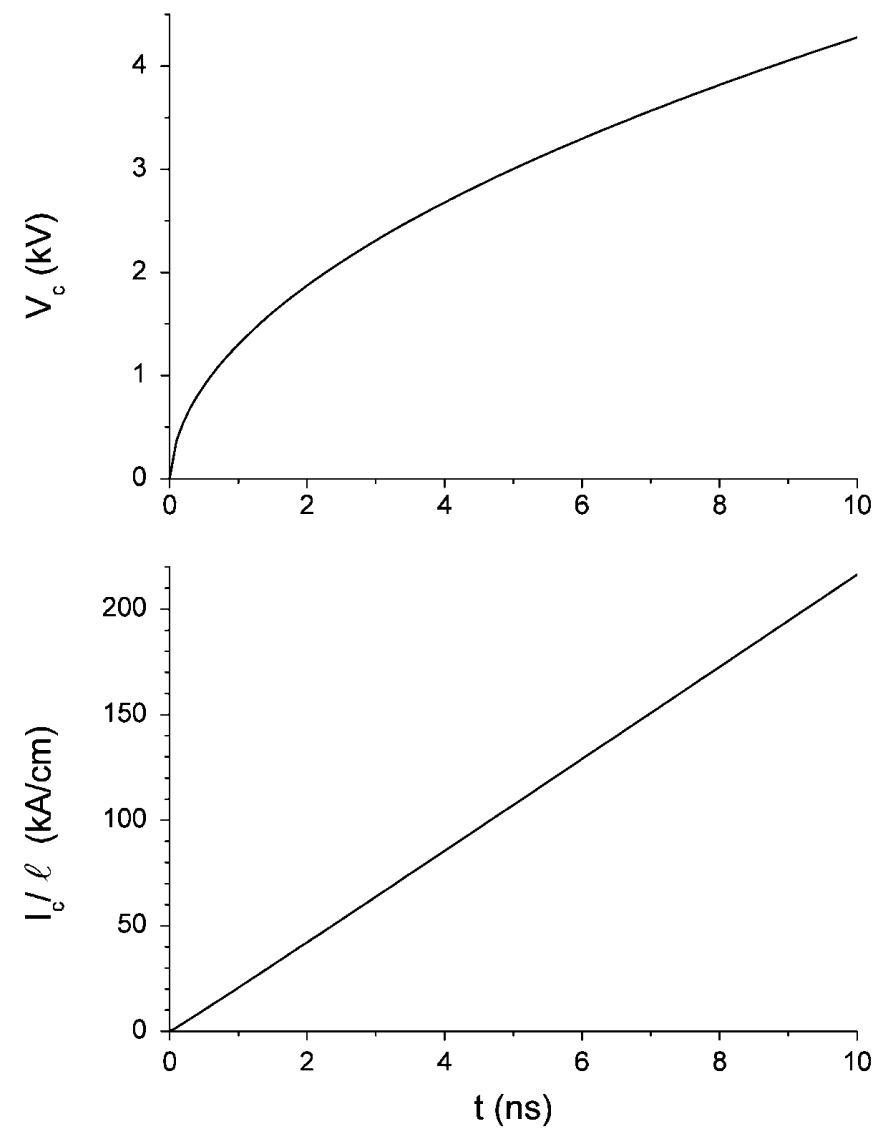

FIG. 2. Voltage and current as a function of time obtained from the evaluation of Eqs. (10) and (11) with $k=1, \mu=80 \mu_{0}, \rho=$ $10^{-7} \Omega \mathrm{m}, \quad h=24 \mu \mathrm{m}, \quad q=500 \mu \mathrm{m}, \quad \delta=50 \mu \mathrm{m}, \quad$ and $\Delta V / t_{0}=10 \mathrm{kV} / \mathrm{ns}$.

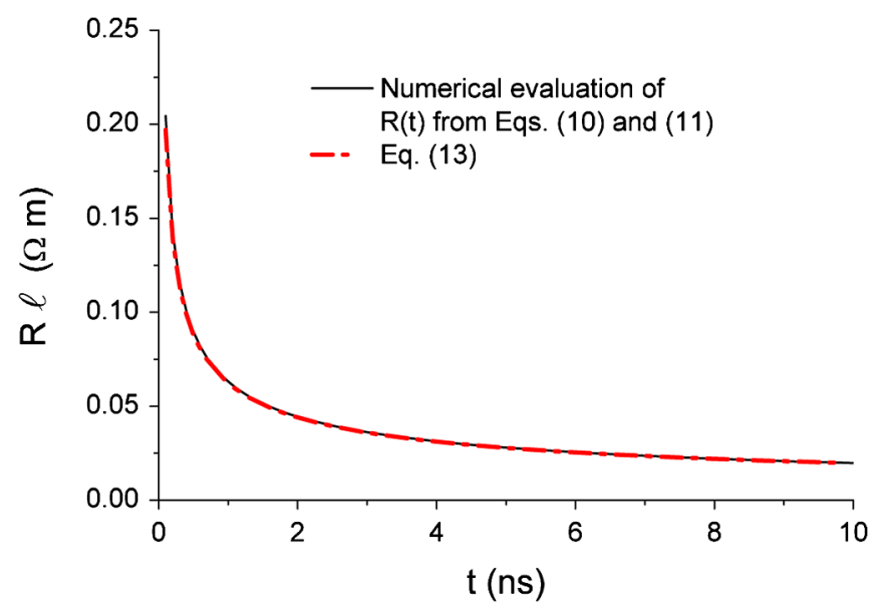

FIG. 3. Resistance (times unit length) as a function of time obtained from the evaluation of Eqs. (10) and (11) (solid black lines) with $\mu=80 \mu_{0}, \rho=10^{-7} \Omega \mathrm{m}, h=24 \mu \mathrm{m}$, $q=500 \mu \mathrm{m}, \delta=50 \mu \mathrm{m}$, and $\Delta V / t_{0}=10 \mathrm{kV} / \mathrm{ns}$. The dashed curve corresponds to Eq. (13) using the same parameters. 

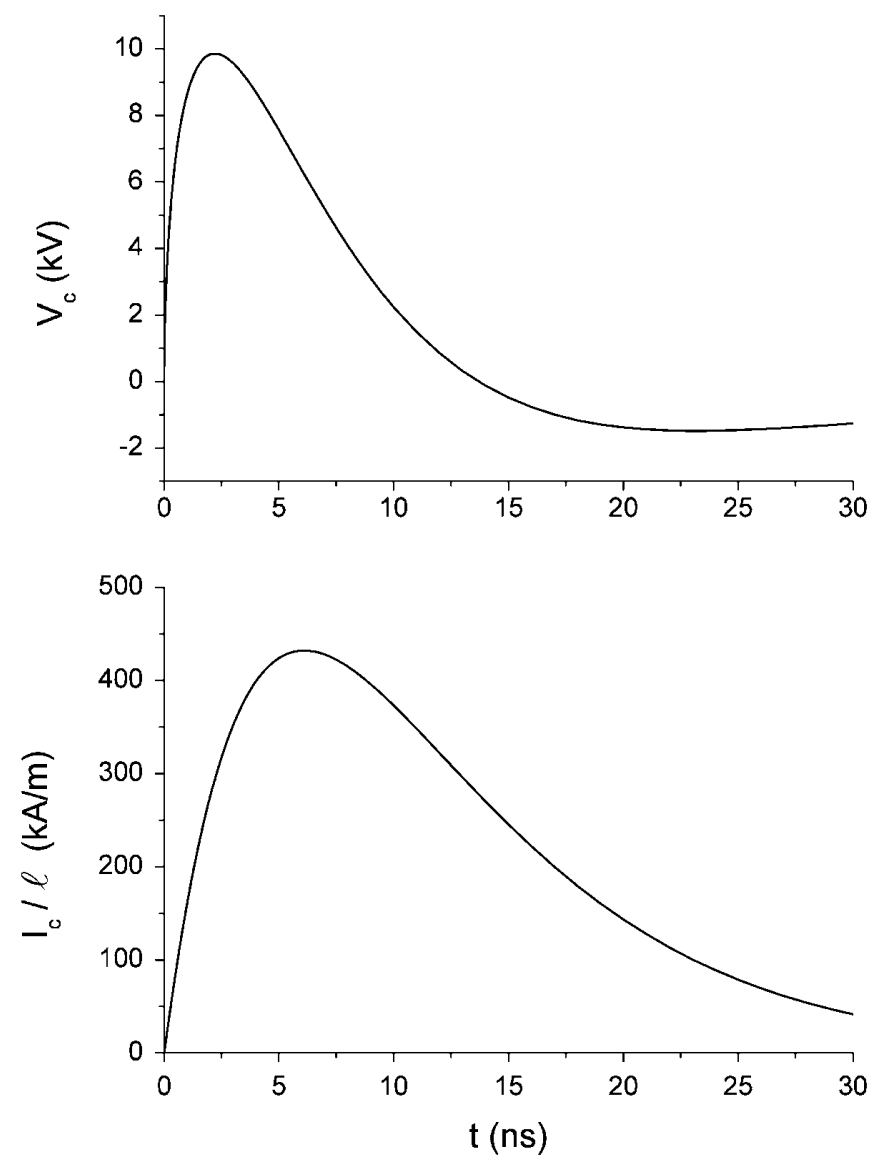

FIG. 4. Voltage and current as a function of time obtained from the evaluation of Eqs. (6) and (7) using the voltage drive time history given by Eq. (14) with $\mu=80 \mu_{0}, \rho=10^{-7} \Omega \mathrm{m}, h=$ $24 \mu \mathrm{m}, q=500 \mu \mathrm{m}, \delta=50 \mu \mathrm{m}, \Delta V / t_{0}=90 \mathrm{kV} / \mathrm{ns}$, and $\alpha=1 / 6 \mathrm{~ns}^{-1}$.

$$
V_{0}(t)=\frac{\Delta V}{t_{0}} t \exp [-\alpha t]
$$

with $\Delta V / t_{0}=90 \mathrm{kV} / \mathrm{ns}$ and $\alpha=1 / 6 \mathrm{~ns}^{-1}$. This choice of drive voltage serves to illustrate the general applicability of the various modeling methods presented in this work, especially when the drive voltage cannot easily be represented in a power law form. The corresponding Laplace transform of Eq. (14) is

$$
\tilde{V}_{0}(s)=\frac{\Delta V}{t_{0}} \frac{1}{(s+\alpha)^{2}} .
$$

Again, the Laplace transforms were obtained from Eqs. (6) and (7) by numerical integration, and results for the core current and voltage are shown in Fig. 4. For this more general voltage drive the approximate expression for $R(t)$ in Eq. (13) is of course no longer applicable.

\section{SIBC FOR FDTD SIMULATION}

A model for the impedance of a ferromagnetic core suitable for implementation in FDTD simulation can be obtained making use of the SIBC formulation of $\mathrm{Oh}$ and Schutt-Aine [15]. A detailed derivation of the SIBC method is given in Ref. [15] and a brief summary of its present implementation in the LSP particle-in-cell simulation code is given below for completeness.

We first note that Eq. (4) may be written in the form

$$
Z_{c}(s)=\frac{2 \sqrt{\mu \rho} A \sqrt{a}}{\ell \delta} \sqrt{\frac{s}{a}} \tanh \left(\sqrt{\frac{s}{a}}\right)=K \sqrt{\frac{s}{a}} \tanh \left(\sqrt{\frac{s}{a}}\right) .
$$

Defining $x=s / a$, we next obtain a fit to the dimensionless function $\sqrt{x} \tanh \sqrt{x}$ in the form

$$
\sqrt{x} \tanh \sqrt{x} \simeq C_{0}\left[1-\sum_{i=1}^{N} \frac{C_{i}}{\omega_{i}+x}\right]
$$

where $C_{0}$ is a constant and $C_{i}$ and $\omega_{i}$ are dimensionless fitting parameters. This form of fitting function implies the following time-domain relationship between the tangential electric and magnetic fields at time $t=m \Delta t$ (with $m$ as a non-negative integer and $\Delta t$ the EM time step) at the termination impedance:

$$
\vec{E}_{t}(m \Delta t)=K^{\prime}\left[\hat{n} \times \vec{H}_{t}\right]-\sum_{i=1}^{N} \vec{A}_{i}(m \Delta t),
$$

where $\hat{n}$ is a unit normal vector and

$$
\begin{aligned}
\vec{A}_{i}(m \Delta t)= & p_{i 1}\left[\hat{n} \times \vec{H}_{t}(m \Delta t)\right]+p_{i 2}\left\{\hat{n} \times \vec{H}_{t}[(m-1) \Delta t]\right\} \\
& +p_{i 3} \vec{A}_{i}[(m-1) \Delta t]
\end{aligned}
$$

with

$p_{i 1}=K^{\prime} \frac{C_{i}}{\omega_{i}}\left[1+\frac{\exp \left(-a \omega_{i} \Delta t\right)-1}{a \omega_{i} \Delta t}\right]$,

$p_{i 2}=K^{\prime} \frac{C_{i}}{\omega_{i}}\left[\frac{1}{a \omega_{i} \Delta t}-\exp \left(-a \omega_{i} \Delta t\right)\left(1+\frac{1}{a \omega_{i} \Delta t}\right)\right]$,

$p_{i 3}=\exp \left(-a \omega_{i} \Delta t\right)$

for $i=1, N$ and

$$
K^{\prime}=C_{0} \frac{2 \sqrt{\mu \rho} A \sqrt{a}}{h \delta},
$$

which follows from Eq. (16). The constants $p_{i 1}, p_{i 2}$, and $p_{i 3}$ are precomputed and the electric field is recursively computed using Eqs. (18) and (19). As discussed in Ref. [15], the recursive procedure is very computationally efficient, requiring only a modest number of additional operations and memory locations.

We obtained a fit to $\sqrt{x} \tanh \sqrt{x}$ over the range $10^{-6} \leq x \leq$ $10^{4}$ using a six-term $(N=6)$ fit and a standard least squares fitting routine [16]. The constant $C_{0}=3.6780451465 \times 10^{2}$ and the fitting parameters $C_{i}$ and $\omega_{i}$ are shown in Table I. (The fitting constants as reported to ten decimal places result in a maximum error of less than $0.1 \%$ over the ten order of magnitude range of the variable $x$.) For the core material parameters and lamination thickness considered here, the 
TABLE I. Dimensionless fitting parameters of Eq. (17) used in the present calculations.

\begin{tabular}{ccc}
\hline \hline$i$ & $C_{i}$ & $\omega_{i}$ \\
\hline 1 & $1.3462950894 \times 10^{-2}$ & $2.4701211709 \times 10^{0}$ \\
2 & $1.6470208581 \times 10^{-1}$ & $2.4308027102 \times 10^{1}$ \\
3 & $1.6977871370 \times 10^{0}$ & $1.1484458284 \times 10^{2}$ \\
4 & $2.1762369282 \times 10^{1}$ & $5.9992961813 \times 10^{2}$ \\
5 & $3.8039022810 \times 10^{2}$ & $3.6713597469 \times 10^{3}$ \\
6 & $4.3068009510 \times 10^{4}$ & $5.1695734919 \times 10^{4}$ \\
\hline
\end{tabular}

frequency parameter $a \simeq 2 \times 10^{6}$ which gives a range for the fit of $2 \leq s \leq 2 \times 10^{10}$. The adequacy of the fit was tested by using the fitting function in the numerical Laplace transform calculations of Sec. III. We found that the fitted impedance function reproduced the voltage and current time histories to better than $1 \%$ after about 20 ps.

\section{CIRCUIT MODEL FOR FERROMAGNETIC CORE}

We show below how a simple circuit model can be deduced from the fit to the core impedance function presented in Sec. IV. The first step is to rewrite Eq. (17) in the following form:

$$
\begin{aligned}
\sqrt{x} \tanh \sqrt{x} & \simeq C_{0}\left[1-\sum_{i=1}^{N} \frac{C_{i}}{\omega_{i}}+\sum_{i=1}^{N} \frac{C_{i}}{\omega_{i}} \frac{\omega_{i}+x}{\omega_{i}+x}-\sum_{i=1}^{N} \frac{C_{i}}{\omega_{i}+x}\right] \\
& =C_{0}\left[1-\sum_{i=1}^{N} \frac{C_{i}}{\omega_{i}}\right]+C_{0} \sum_{i=1}^{N} \frac{\left(C_{i} / \omega_{i}\right) x}{\omega_{i}+x} .
\end{aligned}
$$

We note that since $Z_{c}(0)=0$, the first term on the righthand side of Eq. (22) is zero, and we are left with

$$
Z_{c}(s)=K C_{0} \sum_{i=1}^{N} \frac{\left(C_{i} / \omega_{i}\right) s}{a \omega_{i}+s} .
$$

We now observe that the impedance function is in the form of a series of $N$ circuit elements, each comprised of an inductor and resistor in parallel as illustrated in Fig. 5. The circuit parameters $R_{i}$ and $L_{i}$ are obtained from Eq. (23) and are given by

$$
R_{i}=K C_{0} \frac{C_{i}}{\omega_{i}}
$$

and

$$
L_{i}=K C_{0} \frac{C_{i}}{a \omega_{i}^{2}}
$$

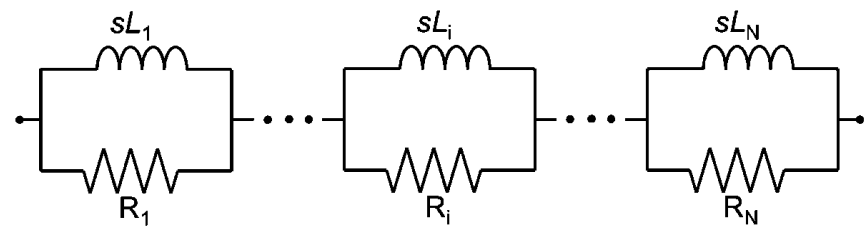

FIG. 5. Equivalent circuit for the ferromagnetic core impedance $Z_{c}(s)$.

\section{COMPARISON OF MODELS AND FDTD SIMULATIONS}

We carried out 2D FDTD simulations of highly idealized ferromagnetic cores to demonstrate the applicability of the impedance models discussed in Secs. II and III. We used the particle-in-cell code LSP [17] to carry out the simulations. An explicit EM field solver was used with uniform $(d x=d y=1 \mu \mathrm{m})$ cell sizes. Two types of simulations were carried out. The configurations for these simulations are illustrated in Fig. 6. In Fig. 6(a), a forward-traveling voltage waveform is injected at the entrance to a parallel plate vacuum transmission line, with a $24 \mu \mathrm{m}$ gap. This transmission line feeds a larger vacuum region containing five individual slabs of material with permeability $\mu>\mu_{0}$, permittivity $\epsilon=\epsilon_{0}$, and finite resistivity $\rho$. The slabs are $50 \mu \mathrm{m}$ thick and $500 \mu \mathrm{m}$ long and assumed to be of infinite extent in the third direction (into and out of the page). Current flows around the rectangular vacuum region and generates magnetic field that diffuses into the individual slabs. This region then looks like a time-dependent impedance to the transmission line. The top and bottom of each slab is capped with a one-cell-thick perfect conductor [indicated by solid black rectangles in Fig. 6(a)] to ensure


FIG. 6. Schematic of the 2D simulation models. In both cases, a forward-traveling voltage waveform is introduced from the left-hand-side feeding a parallel plate vacuum transmission line $24 \mu \mathrm{m}$ in width. In (a), a five slab core is explicitly modeled. The figure indicates the relevant physical dimensions. In (b), the entire core region is replaced by a surface-impedance boundary condition. 
that the magnetic field diffusion in the slabs is $1 \mathrm{D}$, facilitating comparison to the analytic model.

In Fig. 6(b), the entire core region downstream of the parallel plate transmission line is replaced by the SIBC described in Sec. IV. In the examples below, we compare the results of these two simulation models with the numerical solution to the transmission line equations in Sec. III.

For the case of a linear voltage drive with $\Delta V / t_{0}=$ $10 \mathrm{kV} / \mathrm{ns}$, we compare the voltage obtained from an LSP core simulation to evaluation of Eq. (10) for $k=1$ from Fig. 2 with $\mu=80 \mu_{0}$ and $\rho=10^{-7} \Omega \mathrm{m}$. The results are shown in Fig. 7. The simulated voltage has been corrected for the inductance of the vacuum regions in the core that are not explicitly accounted for in the other models. In Fig. 6(a), the impedance of the rectangular current path region enclosing the core slabs is approximately $2.51 \times 10^{-9} \mathrm{H} / \mathrm{m}$. The vacuum space of this region (the area within the current path minus the area occupied by the core slabs) is roughly $37.4 \%$ of the total current path area, or $L_{0}=9.4 \times 10^{-10} \mathrm{H} / \mathrm{m}$. We correct the voltage $V_{m}$ measured in the parallel plate feed region by this inductance value according to $V(t)=V_{m}(t)-L_{0}(d I / d t)$ to arrive at the voltage shown in Fig. 7.


FIG. 7. Voltage as a function of time obtained from the evaluation of Eq. (10) (solid black lines) for the case of a linear voltage drive $(k=1)$ with $\mu=80 \mu_{0}, \rho=10^{-7} \Omega \mathrm{m}, h=$ $24 \mu \mathrm{m}, q=500 \mu \mathrm{m}, \delta=50 \mu \mathrm{m}$, and $\Delta V / t_{0}=10 \mathrm{kV} / \mathrm{ns}$. These results are compared to the results of two different FDTD simulations.
The numerically calculated voltage and current time histories from the model described in Sec. III are in excellent agreement with the FDTD simulation using the SIBC, even at late times ( $>100 \mathrm{~ns})$ where the low frequency behavior of $Z_{c}(s)$ becomes important and the approximation $\tanh (\sqrt{s / a}) \simeq 1$ is no longer valid. We should reiterate here that all our results are based on the assumption of a linear material response and so do not include saturation or hysteresis effects.

At early times in Fig. 7(a), the voltage measured in the transmission line for the simulation using the five individual material slabs is slightly lower than the other results. The finite grid cell size and time step limits the frequencies that can be represented in the core simulation.

We compare the nonlinear voltage drive examples shown in Fig. 4 with results of the two types of FDTD simulations in Fig. 8. Once again, the voltage for the FDTD core simulation shown in Fig. 8(a) has been inductively corrected for the finite vacuum space not included in the transmission line model or the SIBC simulation. The overall agreement between the three curves is very good for the
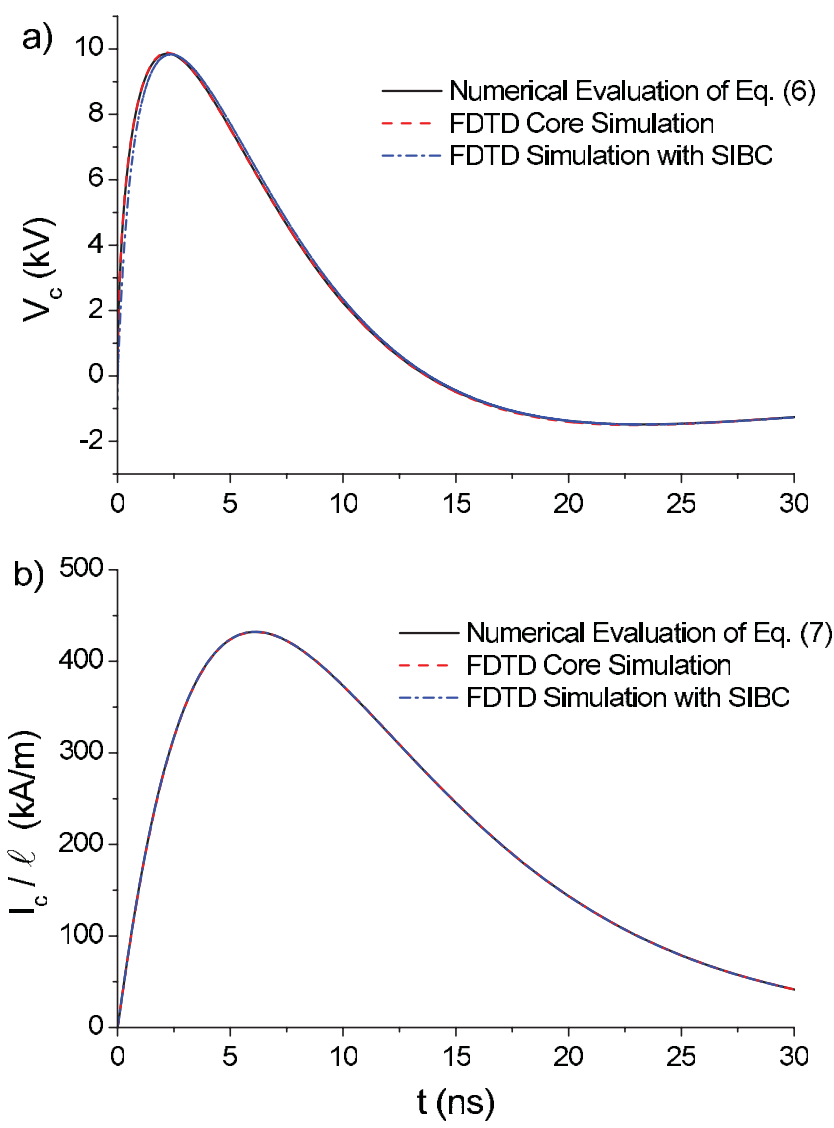

FIG. 8. Voltage and current as a function of time obtained from the evaluation of Eqs. (6) and (7) (solid black lines) for the case of a $t \exp (-\alpha t)$ voltage drive [Eq. (14)] with $\mu=80 \mu_{0}, \rho=$ $10^{-7} \Omega \mathrm{m}, h=24 \mu \mathrm{m}, q=500 \mu \mathrm{m}, \delta=50 \mu \mathrm{m}, \Delta V / t_{0}=$ $90 \mathrm{kV} / \mathrm{ns}$ and $\alpha=1 / 6 \mathrm{~ns}^{-1}$. These results are compared to the results of two different FDTD simulations. 
time scales considered here. The voltage for the FDTD core simulation is slightly less than the numerical solution of Eq. (6) and FDTD simulation using the SIBC over the first two nanoseconds. As for the linear current ramp case shown in Fig. 7, this difference is primarily due to the finite spatial resolution of the FDTD core simulation which does not correctly resolve the skin depth at the highest frequencies. Nevertheless, this nonlinear voltage drive case clearly demonstrates the applicability of the SIBC condition to FDTD simulations of pulsed-power system performance where the dynamic response of the ferromagnetic cores is strongly coupled to the time-dependent evolution of the output power.

Although not shown in Figs. 7 and 8, we have compared these results to the equivalent circuit model for the core described in Sec. V. The circuit calculation results are essentially identical to the SIBC simulation results as expected.

\section{SUMMARY}

Building on the work of Lammeraner and Stafl [12], Grandi et al. [13], and Oh and Schutt-Aine [15], a time-dependent core model for use in the analysis of highcurrent pulsed-power accelerator cavities has been developed. The new model is compared to FDTD simulations of a small, idealized representation of a core composed of five individual ferromagnetic sheets. The use of a relatively small ferromagnetic core in these sample calculations allows the FDTD simulations to resolve (approximately) the magnetic field diffusion into the core bands along with the generation of surface eddy currents along these bands. For the case of a linearly rising current, the new model reproduces the time-dependent core resistance modeled in Ref. [11].

Using newly calculated fitting coefficients, a SIBC previously developed by $\mathrm{Oh}$ and Schutt-Aine [15] allows the complex impedance response of the core to be modeled in a fast and efficient manner without resolving the individual ferromagnetic layers of the core. The SIBC implemented in the LSP code is based on Ref. [15] and also includes a treatment for EM waves of arbitrary incidence angle and polarization [18] at the SIBC (although this feature was not required for the simulations presented here). The SIBC model is found to be in excellent agreement with the transmission line analysis using the effective impedance model of Grandi et al. [13] and the FDTD core simulations.

The main impact of this work will be on future 3D FDTD simulations of pulsed-power accelerators employing multiple LTD cavities. The generality of the SIBC condition to model the time-dependent impedance response of the LTD ferromagnetic cores to arbitrary current waveforms will improve the fidelity of LTD-based accelerator design and performance studies. In addition, we demonstrated that the SIBC can be expressed in terms of an equivalent circuit representation. This formulation should prove useful when incorporated into circuit models of LTD systems [11]. The application of the core impedance model to the analysis of LTD systems will be the subject of a future study.

\section{ACKNOWLEDGMENTS}

The authors would very much like to thank M.K. Matzen, R. McKee, J.L. Porter, and L. Schneider at Sandia National Laboratories for invaluable contributions. Sandia National Laboratories is a multiprogram laboratory operated by Sandia Corporation, a wholly owned subsidiary of Lockheed Martin company, for the U.S. Department of Energy's National Nuclear Security Administration under Contract No. DE-AC04-94AL85000.

[1] B. M. Kovalchuk, V. A. Vizir, A. A. Kim, E. V. Kumpjak, S. V. Loginov, A. N. Bastrikov, V. V. Chervjakov, N. V. Tsou, P. Monjaux, and D. Heut, Russ. Phys. J. 40, 1142 (1997).

[2] A. A. Kim, B. M. Kovalchuk, E. V. Kumpyak, and N. V. Tsoy, Russ. Phys. J. 42, 985 (1999).

[3] A. N. Bastrikov et al., Laser Part. Beams 21, 295 (2003).

[4] M. G. Mazarakis et al., Phys. Rev. ST Accel. Beams 12, 050401 (2009).

[5] A. A. Kim et al., Phys. Rev. ST Accel. Beams 12, 050402 (2009).

[6] L. Chen, L. Zhou, W. Zou, J. Ren, Y. Li, S. Wu, W. Xie, S. Feng, and J. Deng, Acta Phys. Pol. A 115, 1186 (2009).

[7] I. D. Smith, Phys. Rev. ST Accel. Beams 7, 064801 (2004).

[8] S. Humphries, Charged Particle Acceleration (John Wiley, New York, 1986).

[9] W. A. Stygar, M. E. Cuneo, D. I. Headley, H. C. Ives, R. J. Leeper, M. G. Mazarakis, C.L. Olson, J.L. Porter, and T. C. Wagoner, Phys. Rev. ST Accel. Beams 10, 030401 (2007).

[10] W. A. Stygar et al., Phys. Rev. ST Accel. Beams 12, 030402 (2009).

[11] D. V. Rose et al., Phys. Rev. ST Accel. Beams 13, 090401 (2010).

[12] J. Lammeraner and M. Stafl, Eddy Currents (Iliffe Books Ltd., London, 1966).

[13] G. Grandi, M. K. Kazimierczuk, A. Massarini, U. Reggiani, and G. Sancineto, IEEE Trans. Magn. 40, 1839 (2004).

[14] A. A. Kim, M. G. Mazarakis, V. I. Manylov, V. A. Visir, and W. A. Stygar, Phys. Rev. ST Accel. Beams 13, 070401 (2010).

[15] K.S. Oh and J.E. Schutt-Aine, IEEE Trans. Antennas Propag. 43, 660 (1995).

[16] W.H. Press, B.P. Flannery, S. A. Teukolsky, and W. T. Vetterling, Numerical Recipes (Cambridge University Press, Cambridge, 1989).

[17] D. R. Welch, D. V. Rose, M.E. Cuneo, R. B. Campbell, and T. A. Mehlhorn, Phys. Plasmas 13, 063105 (2006).

[18] S. Kellali, B. Jacko, and A. Reineix, IEEE Trans. Electromagn. Compatibility 35, 347 (1993). 